Enferm Bras 2020;19(3):196-201

https://doi.org/10.33233/eb.v19i3.4256

\title{
OPINIÃO \\ Danos psíquicos durante pandemia por COVID-19 no Brasil
}

Marco Orsini*, Jacqueline Stephanie Fernandes do Nascimento**, Marco Antonio Alves Azizi**, Carlos Henrique Melo Reis ${ }^{* *}$, Nicolle dos Santos Moraes Nunes ${ }^{* *}$, Janie Kelly Fernandes do Nascimento**, Julia Fernandes Eigenheer ${ }^{* * * *}$, Adalgiza Mafra Moreno**, Mauricio de Sant'Anna

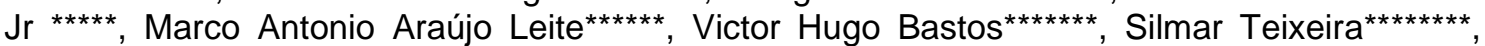
Acary Souza Bulle de Oliveira ${ }^{\star \star \star \star \star \star \star \star \star}$, Cláudia Mendonça Cardoso $0^{\star \star \star \star \star \star \star \star \star \star *}$

*Universidade de Vassouras (USS), Departamento de Medicina, Serviço de Neurologia Universidade Federal Fluminense, ${ }^{* *}$ Universidade Iguaçu, Escola de Medicina, ${ }^{* * *}$ Universidade Iguaçu, Escola de Medicina, Hospital Geral da Posse, Nova Iguaçu, ${ }^{* * * * U n i v e r s i d a d e ~ F e d e r a l ~}$ Fluminense, Departamento de Psiquiatria, ${ }^{* * * *}$ Instituto Federal do Rio de Janeiro, Departamento de Fisioterapia, ${ }^{* * * * * *}$ Serviço de Neurologia, Universidade Federal Fluminense, ****** Universidade Federal do Delta do Parnaíba (UFDPAR), ${ }^{* * * * * * * U n i v e r s i d a d e ~ F e d e r a l ~ d o ~}$

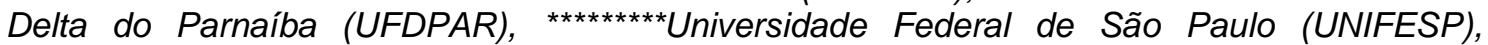
Departamento de Neurologia, ${ }^{* * \star * * * * * *}$ Psicóloga, Universidade do Estado do Rio de Janeiro

Recebido em 27 de maio de 2020; aceito em 17 de junho de 2020.

Correspondência: Jacqueline Stephanie Fernandes do Nascimento, Departamento de Medicina da Universidade Iguaçu, Nova Iguaçu, Rio de Janeiro, Brasil.

Marco Orsini: orsinimarco@hotmail.com

Jacqueline Stephanie Fernandes do Nascimento: jac.fn@hotmail.com

Marco Antonio Alves Azizi: marcoazizi@gmail.com

Carlos Henrique Melo Reis: chmeloreis@hotmail.com

Nicolle dos Santos Moraes Nunes: nicolle.nunes_@hotmail.com.br

Janie Kelly Fernandes do Nascimento: janiekelly@hotmail.com

Julia Fernandes Eigenheer juliafe24@yahoo.com.br

Adalgiza Mafra Moreno dalgizamafra@gmail.com

Mauricio de Sant'Anna Jr: mauricio.junior@ifrj.edu.br

Marco Antonio Araújo Leite maaraujoleite@yahoo.com.br

Victor Hugo Bastos: victorhugobastos@ufpi.edu.br

Silmar Teixeira: silmar_teixeira@yahoo.com.br

Acary Souza Bulle de Öliveira: acary.bulle@unifesp.br

Cláudia Mendonça Cardoso mendonçac@yahoo.com

\section{Resumo}

Introdução: Em 11 de março de 2020 a COVID-19 foi caracterizada pela Organização Mundial de Saúde (OMS) como uma pandemia atrelada a diversos transtornos psíquicos, relacionados à impotência, ao medo, ao pânico e, principalmente, à falta de organização política e de liderança. Métodos: A proposta do presente artigo foi, com base na literatura vigente, atualizar e produzir discussões provocativas acerca do atual contexto de pandemia. Para tal, foi realizada uma busca nas principais bases de dados Lilacs, Bireme e Pubmed, nos idiomas português e inglês, de artigos compreendidos no ano vigente. A escolha ocorreu de forma aleatória, obviamente, seguindo uma linha de reflexão. As palavras-chave pesquisadas foram: COVID-19, saúde mental, pandemia, SARS-COVID-19, Brasil. Discussão: Além dos males à integridade física, a COVID19 também traz prejuízos desastrosos à saúde mental da população. O isolamento social é a principal medida estabelecida até o momento para retardar a propagação da COVID-19. Contudo, esse se relaciona à exacerbação de distúrbios psiquiátricos pré-existentes e ao desenvolvimento de novos quadros, sobretudo de depressão, ansiedade e estresse pós-traumático (todos relacionados ao aumento do risco de suicídio), além do aumento dos casos de violência doméstica. Conclusão: O prévio controle e prevenção da COVID-19 comporta-se como fator de proteção ou "alentecimento" contra problemas psicológicos de graus variados. Estudos 
evidenciaram que investir em educação em saúde a fim de se otimizar o conhecimento da população, no que se refere a COVID-19, auxilia no gerenciamento de comportamentos "otimistas".

Palavras-chave: saúde mental, COVID-19, pandemia, Brasil.

\begin{abstract}
Psychological damage during the COVID-19 pandemic in Brazil

Introduction: On March 11, 2020, COVID-19 was characterized by the World Health Organization (WHO) as a pandemic linked to several psychological disorders, related to impotence, fear, panic and mainly the lack of political organization and leadership. Methods: The proposal of this article will be, based on the current literature, update and produce provocative discussions about the current pandemic context. To this end, it was carried out a search in the main databases Lilacs, Bireme and Pubmed in English and Portuguese from articles of the current year. The choice was made at random, obviously, following a line of reflection. The keywords searched were: COVID19, mental health, pandemic, SARS-COVID-19, Brazil. Discussion: Besides the harm to physical integrity, COVID-19 also brings disastrous damage to the mental health of the population. Social isolation is the main measure used to date to delay the spread of COVID-19. However, this is a problem related to the exacerbation of pre-existing psychiatric disorders and the development of new conditions, mainly of depression, anxiety and post-traumatic stress (all related to the increased risk of suicide), in addition to the increase in cases of domestic violence. Conclusion: The previous control and prevention of COVID-19 behaves as a protective factor or "retardation" against psychological problems of varying degrees. Studies have shown that investing in health education in order to optimize the knowledge of the population, about COVID-19, helps in the management of "optimistic" behaviors.
\end{abstract}

Keywords: mental health, COVID-19, pandemic, Brazil.

\title{
Resumen \\ Daño psicológico durante la pandemia de COVID-19 en Brasil
}

Introducción: El 11 de marzo de 2020, COVID-19 se caracterizó por la Organización Mundial de la Salud (OMS) como una pandemia vinculada a varios trastornos psicológicos, relacionados con la impotencia, el miedo, el pánico y, principalmente, la falta de organización política y social de liderazgo. Métodos: La propuesta del presente artículo, basada en la literatura actual, actualizará y producirá discusiones provocativas sobre el contexto actual de la pandemia. Con este fin, se realizó una búsqueda en las principales bases de datos Lilacs, Bireme y Pubmed, en portugués e inglés, de artículos incluidos en el año en curso. La elección se hizo al azar, obviamente, siguiendo una línea de reflexión. Las palabras clave buscadas fueron: COVID-19, salud mental, pandemia, SARS-COVID-19, Brasil. Discusión: Además del daño físico, COVID-19 también trae daños desastrosos a la salud mental de la población. El aislamiento social es la principal medida establecida hasta ahora para retrasar la propagación de COVID-19. Sin embargo, esto está relacionado con la exacerbación de trastornos psiquiátricos preexistentes y el desarrollo de nuevas afecciones, especialmente depresión, ansiedad y estrés postraumático (todo relacionado con un mayor riesgo de suicidio), además del aumento de casos de violencia doméstica. Conclusión: El control previo y la prevención de COVID-19 se comporta como un factor protector o "estímulo" contra problemas psicológicos de diversos grados. Los estudios han demostrado que invertir en educación sanitaria para optimizar el conocimiento de la población, con respecto a COVID-19, ayuda en la gestión de comportamientos "optimistas".

Palabras-clave: salud mental, COVID-19, pandemia, Brasil.

Introdução

Ao final de dezembro de 2019, a OMS foi alertada sobre inúmeros pacientes com pneumonia na cidade de Wuhan, província de Hubei - República Popular da China. O mundo estava diante de uma nova cepa de coronavírus, essa ainda não identificada em seres humanos. Sete dias após tal sinalização, o governo chinês confirmou a presença do vírus (SARS-COV-2). Um total de sete coronavírus humanos (HCoVs) já foram isolados: HCoV-229E, HCoV-OC43, HCoV-NL63, HCoV-HKU1, SARS-COV (síndrome respiratória aguda), MERS-COV (síndrome respiratória - Oriente Médio), sendo o mais recente o SARS-CoV-2(COVID-19) [1].

Historicamente é a sexta vez que a Saúde Pública sinaliza por Emergência Internacional. Os episódios anteriores foram nos anos de 2009 (H1N1); 2014 (Poliovírus); 2014 (surto localizado de ebola na África Ocidental); 2016 (Zica vírus no Brasil) e 2018 (novo surto de ebola 
no Congo (República Democrática [2]. Vale frisar a proximidade cronológica desses eventos nos últimos anos.

Embora saibamos que cerca de $80 \%$ dos combalidos se recuperam sem necessidade de internação, a outra parcela necessita de cuidados hospitalares. Em nosso país, como em outros locais do globo, a crença que os idosos eram o principal grupo de risco mudou. Não seguimos o mesmo "modelo" de países Europeus e da China. Indivíduos com comorbidades (hipertensão, obesidade, cardiopatias e lesões pulmonares crônicas, diabetes ou câncer) têm maior risco de adoecerem. Torna-se imperioso ressaltar que o quadro clínico provocado pelo SARS-CoV-2 não é exclusivamente pulmonar - trata-se de uma doença inflamatória sistêmica [3]. Cabe mencionar também a atuação de fatores sociais, econômicos, políticos, culturais e educacionais preexistentes ao desenvolvimento da doença.

A eclosão de problemas de saúde mental durante uma pandemia é esperada, contudo, até o presente momento, esse tópico de estudo é escasso em nosso país por parte dos formadores de opinião pública, governantes e pesquisadores. Sendo assim, torna-se primordial essa discussão para auxiliarmo-nos no planejamento, desenvolvimento e assistência à nossa população [4].

\section{Discussão}

Além dos males à integridade física, a COVID-19 também carreia prejuízos à saúde mental da população. Após corroborar o modo de transmissibilidade pessoa-pessoa, em 20 de janeiro, equipes médicas chinesas foram diagnosticadas como infectadas pelo vírus com extrema facilidade e vulnerabilidade - sem um total conhecimento das formas de contaminação $[5,6]$.

Pouco ainda se sabe sobre a COVID-19 (SARS-CoV-2), no entanto, a falta ou demora de assistência médica pode resultar em altas taxas de óbito. Tal fato torna-se importante, visto que sentimentos negativos exacerbados podem desencadear a eclosão de sintomas nãoorgânicos [7]. Isso ocorre, pois o perigo e ameaça de adoecimento impactam diretamente na saúde mental. Pesquisas realizadas com estudantes chineses evidenciaram manifestações psíquicas quanto à real eficiência das providências governamentais de prevenção e controle da COVID-19, aos afazeres diários nos estudos e, especialmente, à real ameaça à própria vida. Esse fato totalizava $63 \%$ dos entrevistados e se tornou fator precipitante para "somatização" de sintomas e de seus relevantes desdobramentos subjetivos na mente de cada indivíduo. Diversos foram os problemas apresentados pelos pacientes, no entanto, alguns similares aos da própria infecção por SARS-CoV-2, como as manifestações gastrointestinais (diarreia, vômitos, náuseas), sendo capazes de "simular" uma possível infecção, intensificando os problemas de saúde mental $[7,8]$.

No Brasil, assim como em outros países do globo, o isolamento social é a principal medida estabelecida visando retardar a propagação da COVID-19 (SARS-CoV-2). Em contrapartida, o distanciamento está relacionado à exacerbação de distúrbios psiquiátricos preexistentes e desenvolvimento de novos quadros, sobretudo de depressão, ansiedade e estresse pós-traumático (todos relacionados ao aumento do risco de suicídio) [9]. O desemprego e o nadir financeiro também são fatores de risco bem reconhecidos como agravantes. Além disso, ocorreu um aumento da violência doméstica e do consumo de álcoolldrogas por parte da população. Vale ressaltar que o impacto da pandemia sobre os pacientes com ideações suicidas pode ser pior em situações de luto e em ambientes com recursos precários, onde o ônus financeiro tende a ser agravado por medidas econômicas pífias de suporte $[9,10]$.

A violência familiar, a qual a população está diariamente exposta, consiste em condutas ameaçadoras ou atitudes agressivas no ciclo familiar, que podem ser físicas, econômicas, psicológicas, além de abuso sexual de crianças, mulheres e agressões entre parceiros [11-13]. Em virtude de as contenções efetuadas a fim de se promover o isolamento social em todo 0 mundo, os indivíduos que sobrevivem em condições voláteis de agressividade familiar estão restringidos às suas residências. Embora necessário, 0 isolamento também exacerba a vulnerabilidade pessoal e coletiva $[12,13]$.

Um estudo realizado por Campbell (2020) evidenciou que esse padrão ocorre de maneira repetida em diversos lugares no mundo, como Estados Unidos, China, Austrália e Brasil. Casos de violência familiar expandiram a partir da determinação de isolamento social e quarentena [14].

$\mathrm{Na}$ ocasião em que a Organização Mundial da Saúde (OMS) decretou situação de pandemia, em 11 de março, o Brasil já contava 52 casos confirmados [15,16]. Desde então, foram divulgadas na mídia divergências entre os pronunciamentos do Presidente da República 
e do Ministro da Saúde, tornando as condutas nacionais relacionadas ao cenário pandêmico extremamente politizadas e ambivalentes. A instabilidade governamental atual se tornou dissipadora do medo, da ansiedade, do descuido e da normatização de ódio; influenciando não unicamente nas respostas emocionais às condições atuais, mas também precipitando o agravamento das dificuldades emocionais e das doenças psiquiátricas preexistentes $[17,18]$.

Devido ao avanço exponencial da doença, profissionais atuantes na linha de frente aumentaram suas cargas de trabalho, contabilizando até 16 horas/dia, impossibilitando-os de concluir um período de sono adequado [19].

Pesquisas anteriores revelaram que, durante a epidemia da Síndrome respiratória aguda grave humana (SARS), profissionais de saúde e sobreviventes foram combalidos por problemas de saúde mental, demonstrando disfunções psicológicas como, por exemplo, o estresse póstraumático e os transtornos depressivos. Assim, fundamentados nas experiências vivenciadas na SARS, acredita-se que quadros clínicos psicológicos semelhantes já eclodem durante a pandemia de COVID-19 [20-22].

Uma pesquisa realizada no Lancet Psychiatry demonstrou que os níveis de depressão, ansiedade, insônia e sintomas de estresse, nas equipes médicas atuantes no combate a epidemias eram $50,7 \%, 44,7 \%, 36,1 \%$ e $73,4 \%$, respectivamente. Outro estudo, realizado nas equipes médicas da cidade de Wuhan, revelou que a intensidade do pânico e da ansiedade estavam associadas ao estresse em curto prazo. Chama a atenção que os enfermeiros apresentavam mais problemas de saúde mental quando comparados aos demais profissionais da saúde [23].

A literatura sinaliza que os profissionais de saúde, principalmente aqueles que atuam em unidades de emergência (enfermarias de unidades de terapia intensiva), possuem maior probabilidade de desenvolverem quadros psiquiátricos desfavoráveis, especialmente quando associados à precariedade do sistema de saúde e das condições de trabalho ofertadas. Apesar dos efeitos do SARS-CoV2 na saúde mental ainda não terem sido estudados com minúcia, é necessário estarmos "vigilantes" sobre o estado psicológico dos indivíduos no decorrer da pandemia vigente. O profissional de saúde representa a primeira linha de defesa ao enfrentamento da pandemia contra a COVID-19 e sua saúde mental não deve ser negligenciada [24].

Mediações oportunas como, por exemplo, suporte psicológico e médico específico são extremamente importantes nesse caótico cenário. $\mathrm{O}$ controle da exposição às informações midiáticas quanto a COVID-19, priorizando informações de cunho científico é também um ponto nevrálgico. Tentativas de manter rotinas diárias de trabalho além de horários regulares de sono e exercícios físicos fazem parte do contexto de saúde mental [25].

Conclusão

O prévio controle e prevenção da COVID-19 comporta-se como fator de proteção ou "alentecimento" contra problemas psicológicos de graus variados. Estudos evidenciaram que investir em educação em saúde a fim de se otimizar o conhecimento da população, no que se refere a COVID-19, auxilia no gerenciamento de comportamentos "otimistas" [26,27]. Ademais, representantes políticos devem arquitetar e fornecer políticas públicas de amparo e assistência aos profissionais de saúde e a sua população como um todo, resguardando o direito a vida, a fim de estabelecer um ambiente favorável a construção de resiliência e de cooperação em um cenário de pandemia [28] e de despertar um sentimento de confiança e segurança nas instituições científicas e governamentais.

Referências

1. Brasil. Ministério da Saúde. Coronavírus COVID-19. [citado 2020 maio 20]. Disponível em: https://coronavirus.saude.gov.br/

2. Organização Pan-Americana de Saúde. Folha informativa - COVID-19 (doença causada pelo novo coronavírus). 2020. [citado 2020 maio 22]. Disponível em: https://www.paho.org/bra/index.php?option=com content\&view=article\&id=6101: covid1 9\&ltemid $=875 /$

3. Usuzaki T, Chiba S, Shimoyama M. How can we evaluate an interrelation of symptoms? Arch Gerontol Geriatr 2020;7:89. https://doi.org/10.1016/..archger.2020.104096 
4. Ransing R, Adiukwu F, Pereira-Sanchez V, Ramalho R, Orsolini L, Teixeira ALS et al. Mental Health Interventions during the COVID-19 Pandemic: a conceptual framework by early career psychiatrists. Asian J Psychiatr 2020;14:51. https://doi.org/10.1016/i.ajp.2020.102085

5. Xinhuanet. The Latest: China confirms human-to-human transmission of 2019-nCoV infections among medical staff. [citado 2020 Maio 21]. http://www.xinhuanet.com/english/2020-01/21/c 138722888.htm

6. Huang Y, Zhao N. Generalized anxiety disorder, depressive symptoms and sleep quality during COVID-19 outbreak in China: a web-based cross-sectional survey. Psychiatry Research 2020;288. https://doi.org/10.1016/j.psychres.2020.112954

7. Liu S, Liu Y, Liu Y. Somatic symptoms and concern regarding COVID-19 among Chinese college and primary school students: A cross-sectional survey. Psychiatry Research 2020;289. https://doi.org/10.1016/i.psychres.2020.113070

8. National Health Commission of the People's Republic of China. Handbook of prevention and treatment of the pneumonia caused by the novel coronavirus (2019-nCoV). 2020. [citado 2020 Maio 23]. http://www.nhc.gov.cn/yzygj/s7653p/202003/46c9294a7dfe4cef80dc7f5912eb1989.sht $\mathrm{ml}$

9. Berk M, Vieta E, Dean OM. Suicide risk and prevention during the COVID-19 pandemic. The Lancet Psychiatry 2020;7(6):468-71. https://doi.org/10.1016/ S22150366(20)30171-1

10. Stuckler D, Basu S, Suhrcke M, Coutts A, Mckee M. The public health effect of economic crises and alternative policy responses in Europe: an empirical analysis. Lancet 2009;374:315-23. https://doi.org/10.1016/S0140-6736(09)61124-7

11. Peterman A, Potts A, O'Donnell M, Thompson K, Shah N, Oertelt-prigione S et al. Pandemics and violence against women and children. Center for Global Development Working Paper 528; 2020. https://www.cgdev.org/publication/pandemics-and-violenceagainst-women-and-children

12. Usher K, Bhulla N, Durkin J, Gyamfi N, Jackson D. Family violence and COVID-19: Increased vulnerability and reduced options for support. Int J Mental Health Nurs 2020. https://doi.org/10.1111/inm.12735

13. Van Gelder N, Peterman A, Potts A, O'Donnell M, Thompson K, Shah N, et al. COVID19: Reducing the risk of infection might increase the risk of intimate partner violence. $\mathrm{E}$ Clinical Medicine Retrieved. 2020. https://doi.org/10.1016/j.eclinm.2020.10034

14. Campbell AM. An increasing risk of family violence during the covid-19 pandemic: strengthening community collaborations to save lives. Forensic Science International: Reports. 2020. https://doi.org/10.1016/j.fsir.2020.100089

15. Reis I. A retórica da crise: democracia e estabilidade institucional no Brasil em tempos da pandemia de coronavírus. Revista NAU Social 2020;11(20):145-55. [citado 2020 Maio 21]. https://cienciasmedicasbiologicas.ufba.br/index.php/nausocial/article/view/36545/21019

16. OMS declara pandemia; em 15 dias, país pode ter 4.000 casos. Folha de São Paulo. 2020, A1. [citado 2020 Maio 22]. Disponível em: http://edicaodigital.folha.uol.com.br/?done=https://acervo.folha.com.br

17. Santos CF. Reflections about the impact of the SARS-COV-2/COVID-19 pandemic on mental health. Braz J Psychiatry 2020;42(3):329-32. https://doi.org/10.1590/1516-44462020-0981

18. Lima CK, Carvalho PM, Lima IA, Nunes JV, Saraiva JS, Souza RI et al. The emotional impact of Coronavirus 2019-nCoV (new Coronavirus disease). Coronavírus. Psiquiatry Res 2020;287. https://doi.org/10.1016/i.psychres.2020.112915

19. The Japan Times. Japanese official looking after Wuhan returnees found dead. 2020. [citado 2020 Feb 2]. https://www.japantimes.co.jp/news/2020/02/02/national/crimelegal/japanese-official-looking-wuhan-returnees-found-dead/

20. Lee AM, Wong JG, Mcalonan GM, Cheung V, Cheung C, Sham PC et al. Stress and psychological distress among SARS survivors 1 year after the outbreak. Can J Psychiatryv 2007;52:233-40. https://doi.org/10.1177/070674370705200405

21. Lu YC, Shu BC, Chang YY, Lung FW. The mental health of hospital workers dealing with severe acute respiratory syndrome. Psychother Psychosom 2006;75:370-5. https://doi.org/10.1159/000095443 
22. Mcalonan GM, Lee AM, Cheung V, Cheung C, Tsang KW, Sham PC et al. Immediate and sustained psychological impacto fan emerging infectious disease outbreak on healthcare workers. Can J Psychiatry 2007;52:241-7. https://doi.org/10.1177/070674370705200406

23. Wu W, Zhang Y, Wang P, Zhang L, Wang G, Lei G et al. Psychological stress of medical staffs during outbreak of COVID-19 and adjustment strategy. J Med Virol 2020. https://doi.org/10.1002/jmv.25914

24. Naushad VA, Bierens JJ, Nishan KP, Firjeeth CP, Mohammad OH, Maliyakkal AM et al. A systematic review of the impact of disaster on the mental health of medical responders. Prehospital Disaster Med 2019;34:632-43. https://doi.org/10.1017/S1049023X19004874

25. Grein TW, Kamara KBO, Rodier G, Plant AJ, Bovier P, Ryan MJ et al. Rumors of disease in the global village: Outbreak verification. Emerg Infect Dis 2000;6:97-102. https://doi.org/10.3201/eid0602.000201

26. Chen $Y$, Jin YL, Zhu LJ, Fang, ZM, Wu N, Du MX et al. The network investigation on knowledge, atitude and practice about COVID-19 of the residents in Anhui Province. Zhonghua Yu Fang Yi Xue Za Zhi 2020;54(4):367-73. ID:mdl-32268643

27. Zhong BL, Luo W, Li HM, Zhang QQ, Liu XG, Li WT et al. 2020. Knowledge, attitudes, and practices towards COVID-19 among Chinese residents during the rapid rise period of the COVID-19outbreak: a quick online cross-sectional survey. Int J Biol Sci 2020;16(10):1745-52. https://doi.org/10.7150/iibs.45221

28. Zhang Y, Ma ZF, 2020. Impact of the COVID-19 pandemic on mental health and quality of life among local residents in Liaoning Province, China: a cross-sectional study. Int J Environ Res Public Health 2020;17. https://doi.org/10.3390/ijerph17072381 